\title{
Automatic Extraction of Rules for Anaphora Resolution of Japanese Zero Pronouns from Aligned Sentence Pairs
}

\author{
Hiromi Nakaiwa \\ NTT Communication Science Laboratories \\ 1-1 Hikarinooka, Yokosuka-shi, \\ Kanagawa-ken, 239 JAPAN \\ nakaiwa@cslab.kecl.ntt.co.jp
}

\begin{abstract}
This paper proposes a method to extract rules for anaphora resolution of Japanese zero pronouns from aligned sentence pairs. The method focuses on the characteristics of Japanese and English in which both the language families and the distribution of zero pronouns are very different. In this method, zero pronouns in the Japanese sentence and the English translation equivalents of their antecedents are extracted from Japanese and English aligned sentence pairs. Then resolution rules for Japanese zero pronouns are automatically extracted using the pairs of Japanese zero pronouns and translation equivalents of their antecedents in English and equivalent word/phrase pairs which were extracted from the aligned sentence pairs, based on the syntactic and semantic structure of the Japanese sentence. This method was implemented in the Japanese-to-English machine translation system, ALT-J/E. The evaluation showed that, for 371 zero pronouns with deictic reference in a sentence set for the evaluation of Japaneseto-English machine translation systems, the rules which were created automatically from Japanese and English aligned sentence pairs correctly resolved $99.2 \%$ of zero pronouns in a window test and $87.6 \%$ of zero pronouns in a blind test.
\end{abstract}

\section{Motivation}

In all natural language, elements that can be easily deduced by the reader are frequently omitted from expressions in texts (Kuno, 1978). This phenomenon causes considerable problems in natural language processing systems. For example, in a machine translation system, the system needs to recognize that elements which are not present in the source language, may become mandatory elements in the target language. In particular, the subject and object are often omitted in Japanese whereas they are often mandatory in English. Thus, in Japaneseto-English machine translation systems, it is necessary to identify case elements omitted from the original Japanese ("zero pronouns") for their translation into English expressions.

Several methods have been proposed with regard to this problem (Kameyama, 1986; Walker et al., 1990; Yoshimoto, 1988; Dousaka, 1994). When considering the application of these methods to a practical machine translation system for which the translation target area cannot be limited, it is not possible to apply them directly, both because their precision of resolution is low as they only use limited information, and because the volume of knowledge that must be prepared beforehand is so large.

To overcome these kinds of problems, several methods to resolve zero pronouns which consider applications for a practical machine translation system with an unlimited translation target area, have been proposed (Nakaiwa and Ikehara, 1992; Nakaiwa and Ikehara, 1995; Nakaiwa and Ikehara, 1996). These methods use categorized semantic and pragmatic constraints such as verbal semantic attributes (Nakaiwa et al., 1994) and types of modal expressions and conjunctions as a condition for anaphora resolution of zero pronouns and determine antecedents of zero pronouns depending on the typical category of three types of semantic and pragmatic constraints.

However, with these methods it is necessary to make resolution rules for zero pronouns by hand. So, to make robust rules with wide coverage takes a lot of time and labor and analysts who make these resolution rules must be familiar with the NLP system itself. Furthermore, the types of zero pronouns change depending on the types of documents which must be analyzed. So, resolution rules must be made depending on the target domain of the documents. But, it is very difficult to make rules for every domain because of the time consuming labor and the need for expertise. Because of these problems, a method to make resolution rules for zero pronouns effectively and efficiently is greatly needed. 
In order to acquire resolution rules for a NLP system effectively and efficiently, various methods have been proposed. One typical method for this purpose is to use a corpus for extracting resolution rules by analyzing each sentence in the corpus. With regard to the automatic extraction of resolution rules for zero pronouns, several methods have been proposed (Murata and Nagao, 1997; Nasukawa, 1996). But these methods only use monolingual corpora and they find it difficult to extract resolution rules for zero pronouns whose referents are normally unexpressed in Japanese. Furthermore, rules can only be made when similar expressions to those containing the zero pronouns are found in the corpus.

In order to take into account the kinds of problems which are caused by monolingual corpora, it seems that a bilingual corpus consisting of pairs of a sentence in one language and a translation of the sentence is better than a monolingual corpus for the purpose of acquiring resolution rules for zero pronouns. This is particularly so with a bilingual corpus of Japanese and English whose language families are so different and in which the distribution of zero pronouns is also very different. This combination is more useful than the bilingual corpora of language pairs whose language families are similar.

Several methods have been proposed with regard to acquiring various kinds of rules such as translation rules, grammar rules, dictionary entries and so on from bilingual corpora (Dagan et al., 1991; Dagan and Church, 1994; Fung and Church, 1994; Tanaka, 1994; Yamada et al., 1995). From the point of view of the extraction of resolution rules for zero pronouns, a technique to extract zero pronouns in a sentence in one language and translation equivalents of their antecedents in a translation from aligned sentence pairs is needed. Such a technique has recently been proposed, and a method to extract Japanese zero pronouns in Japanese sentences and translation equivalents of their antecedents in English sentences from aligned sentence pairs has been developed. (Nakaiwa and Yamada, 1997). Another technique which is needed, is to make rules automatically to resolve zero pronouns using pairs of equivalent words/phrases with zero pronouns and their antecedents from aligned sentence pairs. Several methods to extract rules and dictionary entries for machine translation have been proposed such as (Yamada et al., 1995). But there is currently no proposed method for extracting resolution rules for zero pronouns automatically using bilingual corpora.

In this paper, I propose a widely applicable method to extract resolution rules for Japanese zero pronouns from Japanese and English aligned sentence pairs automatically using pairs of equivalent words/phrases and pairs of zero pronouns and their antecedents.

\section{Appearance of Zero Pronouns and Their Antecedents within Japanese and English Aligned Sentence Pairs}

In order to understand the distribution of zero pronouns with antecedents within Japanese and English aligned sentence pairs, in this section, I examine which zero pronouns in Japanese must be explicitly translated into English and where their translation equivalents in English appear, using a test set designed to evaluate the performance of Japanese-toEnglish machine translation systems (Ikehara et al., 1994). The test set (3718 sentences) has many examples of zero pronouns making intrasentential and deictic references. The sentence set was created to test the coverage of expressions that can be translated by Japanese to English MT systems based on the varieties of Japanese expressions and the differences between Japanese and English. The sentence set has approximately 500 kinds of test items. Each sentence has a manual translation, and almost all of the sentences can be translated without contextual information ( 3704 sentences out of 3718 sentences). A MT system can be evaluated by comparing its output to the equivalent manual translation. Each sentence is expressed in natural Japanese and the sentence set covers many different expressions.

This is an example of a zero pronoun in Japanese whose referent is expressed in the English translation

(1) $(\phi-g a) \begin{gathered}\text { hon-wo } \\ \text { book-oBJ }\end{gathered} \begin{gathered}\text { yomi-tai } \\ \text { read-WANT-To }\end{gathered}$

I want to read a book.

In this expression, the Japanese sentence contains the modal expression tai which indicates HOPE. This modal expression causes the default referent of the subject zero pronoun to be "writer" or "speaker" which is translated as " $\mathrm{I}$ " in English.

The results of the examination of zero pronouns and their referential elements in the functional test sentence set are shown in Table 1 . There were a total of 525 zero pronouns in 463 sentences. The location of the referential elements can be divided into 2 kinds: those expressed in the same sentence, and those not expressed in the same sentence. The latter were further classified into 6 kinds.

- The zero pronoun is not translated because the passive voice is used.

- The referent is the writer or speaker, "I" or a group, "we".

- The referent is the reader or hearer, "you".

- The referent is human but it is not known who the human is.

- The zero pronoun should be translated as "it".

- The referent is another specific element. 
According to this study of the functional test set, in 371 out of 525 instances $(71 \%)$ the referent was not expressed in the sentence. Of these, the zero pronouns could be left unexpressed by converting the translation to the passive voice in 156 instances $(30 \%)$. The other zero pronouns, 215 instances $(41 \%)$, referred to referents that did not appear in the Japanese sentence but appear in the English translation. This result shows that aligned sentence pairs will be effective for extracting zero pronouns and their antecedents automatically by determining zero pronouns in Japanese and translation equivalents of their antecedents in English.

According to a further examination of the English equivalents of Japanese zero pronouns in the sentence set in Table 1, depending on the types of referential elements, the style of the English equivalents are different. These characteristics can be summarized in the following:

1. Deictic referents in English (215 instances)

These elements are often translated as personal pronouns such as "I" or "you" or indefinite "one".

2. Anaphoric antecedents in English (154 instances; intrasentential)

These elements are often translated as personal pronouns, demonstratives such as "that", definite noun phrases such as a noun phrase with definite article (e.g. "the company") or anaphoric "one".

English expressions of these two types can be preferred candidates for translation equivalents of Japanese zero pronouns.

Furthermore, according to an analysis of these aligned sentence pairs (Nakaiwa and Ikehara, 1995; Nakaiwa and Ikehara, 1996), zero pronouns in the corpus can be successfully resolved using three kinds of semantic and syntactic constraints: verbal semantic attributes, the types of modal expressions and conjunctions. So, for making suitable rules for resolving Japanese zero pronouns in aligned sentence pairs, the use of these semantic and pragmatic categories which are extracted from the syntactic and semantic structure of the Japanese sentence in aligned sentence pairs, will be effective.

\section{A Method for Extraction of Resolution Rules for Japanese Zero Pronouns}

This section describes a method for automatically extracting resolution rules for Japanese zero pronouns from Japanese and English aligned sentence pairs. Figure 1 shows an overview of the system. As shown in this figure, the Japanese and English sentences within the aligned sentence pairs are analyzed separately by Japanese and English syntactic and semantic parsers. Next, the system extracts the pairs of Japanese word/phrase and their English equivalent word/phrase, by comparing these two structures, based on the Japanese syntactic and semantic structures and the English syntactic and semantic structures which are created by the Japanese and English parsers. Then, based on the characteristics of the translation equivalents of antecedents of Japanese zero pronouns in English, which was discussed in Section 2, Japanese zero pronouns in the Japanese sentence and the translation equivalents of their antecedents in the English sentence are extracted. By using these results, based on the Japanese syntactic and semantic structure, the resolution rules for Japanese zero pronouns within Japanese sentences are created. In the next step, the resolution rules are used for the semantic and pragmatic analysis of the Japanese sentence by the Japanese syntactic and semantic parser within the whole rule extraction system. The same Japanese and English aligned sentence pairs are inputted in the system and resolution rules of Japanese zero pronouns are again extracted. These processes are repeated until the system cannot extract any more rules for resolution of Japanese zero pronouns from the aligned sentence pairs.

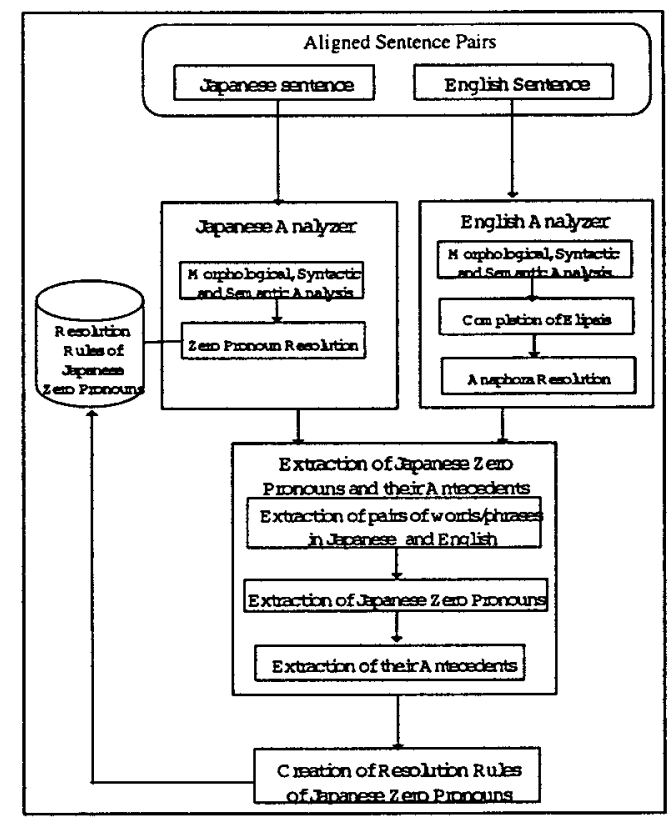

Figure 1: Process for Automatic Extraction of Resolution Rules for Japanese Zero Pronouns

This method has been implemented in the Japanese to English machine translation system. ALT-J/E (Ikehara et al., 1991). The system, which is described in Figure 1, can extract English translation equivalents of Japanese zero pronouns from the aligned sentence pairs. So, these results can be used for the extraction of translation rules for Japanese 


\begin{tabular}{|c|c|c|c|c|c|c|c|c|c|c|c|c|}
\hline \multirow{3}{*}{$\begin{array}{c}\text { Loc. } \\
\text { of } \\
\text { zero } \\
\text { pron. }\end{array}$} & \multicolumn{11}{|c|}{ Loc. of 'referential elements' } & \multirow{3}{*}{$\begin{array}{l}\text { To- } \\
\text { tal }\end{array}$} \\
\hline & \multicolumn{5}{|c|}{ Intrasentential } & \multicolumn{6}{|c|}{ Deictic } & \\
\hline & $h a$ & $g a$ & 0 & ni & misc & Psve & $\begin{array}{c}\mathrm{T} \\
\text { we }\end{array}$ & you & $\begin{array}{l}\text { hu- } \\
\text { man }\end{array}$ & it & $\begin{array}{l}\mathrm{mi} \\
\mathrm{sc}\end{array}$ & \\
\hline$\overline{h a}$ & 1 & 0 & $\overline{0}$ & $\overline{0}$ & $\overline{0}$ & 4 & $\mathbf{0}$ & 2 & $\overline{0}$ & $\mathbf{0}$ & 0 & 7 \\
\hline$g a$ & 119 & 12 & $\overline{0}$ & 2 & 8 & 150 & 85 & 24 & 30 & 51 & $\begin{array}{c}0 \\
0\end{array}$ & 481 \\
\hline$o$ & 4 & 0 & 5 & 1 & 0 & $\mathbf{0}$ & $\begin{array}{l}\mathbf{0} \\
\end{array}$ & 0 & 1 & 7 & $\overline{0}$ & 18 \\
\hline$n i$ & $\overline{1}$ & 0 & $\overline{0}$ & 0 & $\overline{0}$ & 2 & 3 & 8 & 0 & 0 & 1 & 15 \\
\hline no & $\overline{0}$ & 0 & 1 & 0 & $\mathbf{0}$ & 0 & 1 & 0 & 0 & 2 & 0 & 4 \\
\hline Total & \multicolumn{5}{|c|}{154} & \multicolumn{6}{|c|}{371} & 525 \\
\hline
\end{tabular}

Table 1: Distribution of zero pronouns and their referential elements

zero pronouns to English in a Japanese to English machine translation system.

In the next two subsections, I describe the details of the extraction of Japanese zero pronouns in Japanese sentences and translation equivalents of their antecedents in English sentences and the construction of resolution rules for Japanese zero pronouns.

\subsection{Extraction of Japanese Zero Pronouns and Their Antecedents}

The method to extract Japanese zero pronouns and their English equivalents consists of the following steps $^{1}$.

1) Analysis of Japanese and English sentences In this step, I use morphological, syntactic and semantic analyzers of Japanese in ALT-J/E for the analysis of Japanese sentences and Brill's English tagger (Brill, 1992) for the analysis of English sentences ${ }^{2}$.

2) Extraction of the pairs of Japanese word/phrase and their English equivalent word/phrase.

\footnotetext{
${ }^{1}$ For the details of the extraction of Japanese zero pronouns in Japanese sentences and translation equivalents of their antecedents in English equivalent sentences, refer to Nakaiwa and Yamada (1997).

${ }^{2}$ As shown in Figure 1, if the English analyzer can supplement ellipses in English ("I sing a song and $\phi$ play a piano."), then even if translation equivalents of the antecedents of zero pronouns in Japanese are omitted in English sentences, the overall system can determine their an tecedents from the completed elements. Furthermore, when the English analyzer contains an anaphora resolution process such as Lappin and Leass (1994), even if the antecedents of zero pronouns in Japanese are anaphoric expressions such as pronouns and definite noun phrases, the anaphora resolution process determines the antecedents of anaphoric expressions in English and the overall system can determine intersentential and intrasentential resolution rules of Japanese zero pronouns by using extracted pairs of the antecedents of anaphoric expressions in English and their Japanese equivalents. But, for now, I am only using an English tagger as the English analyzer for the primary examination because the extraction of pairs, Step 2, only needs the parts of speech of the English words.
}

In this step, I use the following information: ${ }^{3}$.

- bilingual dictionary for Japanese to English MT system, ALT-J/E

This dictionary is used for the determination of pairs of equivalent word phrases of Japanese and English.

- English dictionary for English generation in ALT-J/E

This dictionary is used when the suffix differs: for example the derivative, 'ing' between an English word in the bilingual dictionary entry and the English word in the English sentence within the aligned sentence pair.

- ignore function words such as prepositions, determiners and others from the English sentence to find Japanese equivalent words/phrases in Japanese

This is because function words must often be changed depending on the types of head such as verb for preposition and noun for determiner in English.

3) Extraction of the candidates for Japanese zero pronouns within the Japanese sentence

In this step, the system extracts Japanese zero pronouns which are determined by syntactic and semantic analysis of Japanese within the syntactic and semantic structure of the Japanese sentence.

4) Extraction of the candidates for translation equivalents of antecedents of Japanese zero pronouns within the English sentence

In this step, the following English words/phrases are extracted from the English sentence as possible translation equivalents ${ }^{4}$ :

\footnotetext{
${ }^{3}$ For details of this step, refer to Yamada et al. (1996).

${ }^{4}$ In this paper, I only extract these 4 types of English words/phrases for the candidates which appeared in the test set and the examination of other types of English words/phrases which can be the candidates of translation equivalents of antecedents of Japanese zero pronouns, such as "they", "he" and "she", remains as future work.
} 
- personal pronouns such as "I" or "you"

- "one".

- demonstratives such as "that"

- definite noun phrases such as a noun phrase with definite article (e.g. "the company")

5) Determination of zero pronouns in Japanese sentences and their referents in English sentences

The pairs of Japanese words/phrases and English equivalent words/phrases and the pairs of zero pronouns in a Japanese sentence and translation equivalents of their antecedents in the English sentence are determined from the candidates for the pairs of Japanese word/phrases and their English equivalent word/phrases which were extracted at step 2, the candidates for Japanese zero pronouns within the Japanese sentence which were extracted at step 3 and the candidates for translation equivalents of antecedents of Japanese zero pronouns within the English sentence which were extracted at step 4. This determination is conducted based on how strongly related the candidates are and how many pairs can be extracted from these candidates.

\subsection{Construction of Resolution Rules for Japanese Zero Pronouns}

Using the extracted Japanese zero pronouns and their antecedents from Japanese and English aligned sentence pairs and the syntactic and semantic structures of Japanese sentences, the system constructs resolution rules for Japanese zero pronouns. For this construction, verbal semantic attributes, the types of modal expressions and conjunctions within Japanese syntactic and semantic structure are used for the resolution conditions of Japanese zero pronouns. In the implementation in ALT-J/E, the rules are extracted using the case type of the zero pronoun, the verbal semantic attributes (VSA; 107 categories) of the verb which governs the zero pronoun (Nakaiwa et al., 1994) and categorized types of modal expression (134 categories) in the unit sentence which contains the zero pronoun (Kawai, 1987) and categorized types of conjunction ( 56 categories) which are directly connected to the unit sentence. For example from aligned sentence pair (1), the rule (3) is extracted from the syntactic and semantic structure (2) in Figure 2.

When zero pronouns, whose resolution conditions are the same but whose antecedents are different, occur within aligned sentence pairs, the resolution rule selects the referent which appears most often as the antecedent of the zero pronouns. For example, when unit sentences with zero pronouns in subject position and with category "A" modal expression occur eight times within aligned sentence pairs and when the extracted antecedents of the zero pronouns are "I" for 5 zero pronouns and "you" for 3 zero pronouns, the resolution rule with the condition of modal expression " $A$ " determines the antecedents of zero pronouns in subject position as " $\mathrm{I}$ ".

\section{Evaluation}

\subsection{Evaluation Method}

The method to extract resolution rules for zero pronouns from aligned sentence pairs which was discussed in Section 3 was evaluated by automatically extracting resolution rules for Japanese zero pronouns from the functional test sentence set which is already aligned, one sentence with one sentence. This evaluation was conducted using the Japanese to English MT system, ALT-J/E for the Japanese analysis and Brill's tagger for the English analysis. The conditions for the evaluation were as follows.

\subsubsection{Evaluation of Target Sentence Pairs}

The evaluation used Japanese and English aligned sentence pairs which contain zero pronouns with deictic references ( 371 instances) in a test set designed to evaluate the performance of Japanese-to-English machine translation systems (Ikehara et al., 1994) (3718 sentence pairs).

\subsubsection{Resolution Rules}

For the sentence pairs which contain zero pronouns with deictic reference, resolution rules for these zero pronouns were extracted by examining verbal semantic attributes, the types of modal expressions and conjunctions within the syntactic and semantic structure of Japanese sentences (Section 3.2 ). In this evaluation, the semantic constraints for cases by verb were not taken into consideration ${ }^{5}$.

\subsubsection{Evaluation Parameters}

To examine the effectiveness of automatically extracting resolution rules for Japanese zero pronouns, I examined the accuracy of resolution rules which are automatically extracted using three kinds of semantic and syntactic constraints: verbal semantic attributes, the types of modal expressions and conjunctions. As a baseline for comparison, I also examined the accuracy of resolution by using rules which only consider the occurrence of antecedents in the same case elements such as subject and object as follows:

- The most frequently occurring antecedent in the same case element is used for the antecedents of zero pronouns in the case element.

- Antecedents in the same case element are proportionally determined with the weight of the

\footnotetext{
${ }^{5}$ If the semantic constraints for cases by verb are taken into consideration, the accuracy of resolution will be better. But, the suitability of the semantic constraints for cases needs to be taken into consideration and this remains as future work.
} 
(2) Syntactic and Semantic Structure of Japanese Sentence (1) and zero pronoun equivalent

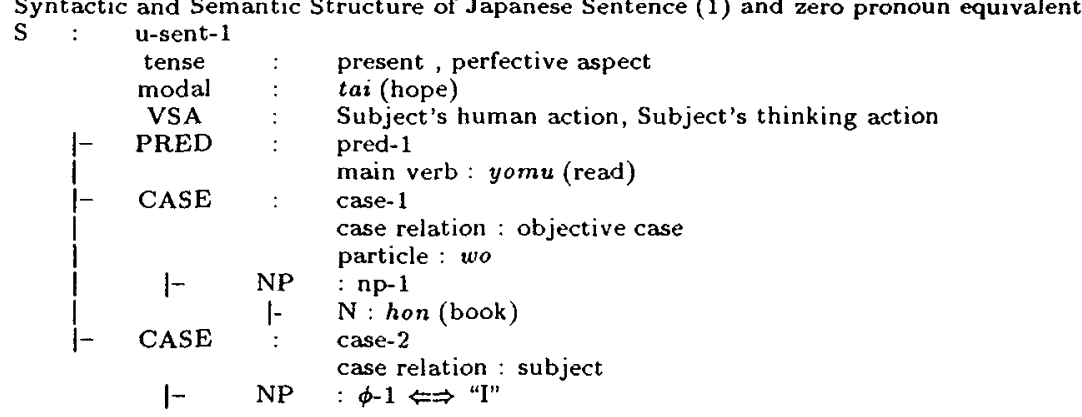

(3) Extracted Resolution Rule for Zero Pronouns in Japanese Sentence (1)

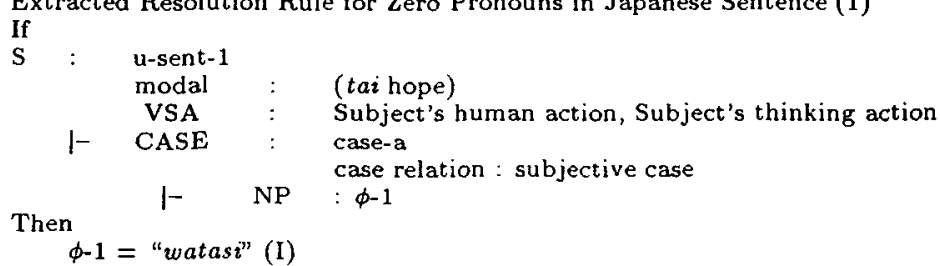

Figure 2: An Example of Syntactic and Semantic Structure and Extracted Resolution Rule

occurrence of the antecedents in the case element.

For example, when the antecedents of zero pronouns in one case element are "I" 3 times and "you" 2 times, the former rule achieves $60 \%(=3 / 5)$ resolution accuracy whilst the latter rule achieves $52 \%(=$ $3 / 5 * 3 / 5+2 / 5 * 2 / 5$ ) resolution accuracy.

The accuracy of resolution is evaluated by the following 2 tests:

(a) window test All zero pronouns with deictic referents (371 instances), which were used for extracting resolution rules, were examined for their accuracy of resolution. As detailed in this paper, I have tried to evaluate the limitations to the accuracy of the rules which are extracted by the method.

(b) blind test 370 zero pronouns out of 371 zero pronouns were used for extracting resolution rules and these rules were applied to the remaining zero pronoun, then the process was repeated 371 times. By calculating the mean of the successfully resolved zero pronouns, the accuracy of resolution was examined. As shown in the next section, I have tried to evaluate the generality of the rules which are extracted by the method.

\subsubsection{Successfully Resolved Zero Pronouns}

When a rule which can determine the antecedent of a zero pronoun is extracted by the method, I judge that the zero pronoun is resolved successfully ${ }^{6}$

\footnotetext{
${ }^{6}$ Because the rules for the evaluation are automatically created from aligned sentence pairs by the method proposed in this paper, it is very difficult to extract only
}

\subsection{Resolution Accuracy}

The resolution accuracy of extracted rules is shown in Table 2. As shown in this table, the accuracy of rules using three kinds of conditions (modal expressions, verbal semantic attributes and conjunctions) is as high as $99.2 \%$ in the window test and $87.6 \%$ even in the blind test. In contrast, resolution rules which only consider the occurrence of antecedents achieve a low resolution accuracy; $46.4 \%$ in the window test and $46.1 \%$ in the blind test for resolution rules using the referent which appears most often and $31.8 \%$ in the window test and $30.6 \%$ in the blind test for resolution rules using the referent which is proportionally determined with the weight of the ocurrence. For the 3 rule sets which are extracted using only one condition each, the order of the resolution accuracy is as follows: modal expression, verbal semantic attributes and conjunctions. Furthermore, if the modal expression and verbal semantic attributes are used together for the creation of resolution rules, the resolution accuracy is as high as $95.2 \%$ in the window test and $81.7 \%$ in the blind test. This shows that the use of these two conditions for the automatic extraction of resolution rules is most effective for the resolution of Japanese zero pronouns with deictic referents. These results demonstrate that this method of automatically creating rules using three kinds of conditions from aligned sentence

the rules which are most suitable for the aligned sentence pairs and the most general rules without using machine learning techniques. In this paper, I only discuss how resolution rules are automatically created and how many zero pronouns can be resolved by using these rules. The method to extract the best rule set remains as future work. 
pairs can correctly resolve almost all Japanese zero pronouns with deictic references ${ }^{7}$.

According to these results, the proposed method is effective for the automatic extraction of resolution rules for Japanese zero pronouns from Japanese and English aligned sentence pairs and by using a large amount of aligned sentence pairs it is possible to extract resolution rules for almost all Japanese zero pronouns.

Table 2: Resolution accuracy for conditions of resolution in automatically created rules

\begin{tabular}{c||c|c}
\hline \multicolumn{1}{c||}{ condition } & \multicolumn{2}{c}{ resolution accuracy } \\
\cline { 2 - 3 } & window test & blind test \\
\hline modal exp. & $74.9 \%$ & $64.2 \%$ \\
& $(278)$ & $(238)$ \\
\hline VSA & $70.9 \%$ & $\mathbf{5 2 . 6 \%}$ \\
& $(263)$ & $(195)$ \\
\hline conjunction & $\mathbf{5 5 . 0 \%}$ & $\mathbf{4 8 . 8 \%}$ \\
& $(\mathbf{2 0 4 )}$ & $(\mathbf{1 8 1 )}$ \\
\hline modal exp. + & $95.2 \%$ & $81.7 \%$ \\
VSA & $(\mathbf{3 5 3})$ & $(\mathbf{3 0 3})$ \\
\hline modal exp. + & $90.3 \%$ & $79.5 \%$ \\
conjunction & $(\mathbf{3 3 5})$ & $(295)$ \\
\hline VSA + & $\mathbf{8 7 . 9 \%}$ & $68.2 \%$ \\
conjunction & $(326)$ & $(253)$ \\
\hline modal exp. + & $99.2 \%$ & $87.6 \%$ \\
VSA + & $(\mathbf{3 6 8})$ & $(\mathbf{3 2 5})$ \\
conjunction & & \\
\hline \hline occurrence & $46.4 \%$ & $46.1 \%$ \\
(most often) & $(172)$ & $(171)$ \\
\hline occurrence & $\mathbf{3 1 . 8 \%}$ & $\mathbf{3 0 . 6 \%}$ \\
(proportionally & $(117.9)$ & $(113.5)$ \\
selected) & & \\
\hline \hline
\end{tabular}

\section{Conclusion}

This paper proposes a powerful method for the extraction of resolution rules for Japanese zero pronouns from Japanese and English aligned sentence pairs. In this paper, I have only discussed Japanese and English language pairs. But this method can be applied to various kinds of language pairs such as Italian and English, and the effectiveness of the extracted rules depends on how different the two languages are. In the future, I will examine methods for extracting the most effective and most general rules for zero pronouns resolution using machine learning techniques. Furthermore, I would like to realize an overall system with an English syntactic and semantic parser and evaluate the effect of the anaphora resolution and omission of ellipsis in English for the

\footnotetext{
${ }^{7}$ This method does not uses any heuristics which were used in other methods of anaphora resolution such as Murata and Nagao (1997) for Japanese nouns and Lappin and Leass (1994) for English pronouns and achieves relatively high accuracy $(87.6 \%)$ even in the blind test. So, if I also use heuristics for the resolution of Japanese zero pronouns, the accuracy will be higher. The examination of the combination of extracted resolution rules and heuristics for the resolution of Japanese zero pronouns remains as future work.
}

extraction of resolution rules for zero pronouns with intersentential and intrasentential antecedents.

\section{Acknowledgments}

I would like to thank Professor Jun'ichi Tsujii for helpful discussion of many of the ideas and proposals presented here during my star at UMIST from September 1995 to September 1996. I am also grateful to several anonymous reviewers of ACL/EACL97 workshop on anaphora for helpful comments on earlier drafts of the paper.

\section{References}

Eric Brill. 1992. A simple rule-based part of speech tagger. In Proc. of ANLP92, pages 152-155, ACL.

Ido Dagan, Alon Itai and Ulrike Schwall. 1991. Two languages are more informative than one. In Proc. of 29th Annual Meeting of $A C L$, pages 130-137, ACL.

Ido Dagan and Kenneth W Church. 1994. Termight: Identifying and translating technical terminology. In Proc. of ANLP94, pages 34-40, ACL.

Kouji Dousaka. 1994. Identifying the Referents if Japanese Zero-Pronouns based on Pragmatic Condition Interpretation. In Trans. of IPS Japan, 35(10):768-778. In Japanese.

Pascale Fung and Kenneth W. Church. 1994. Kvec: A new approach for aligning parallel texts. In Proc. of COLING94, pages 1096-1102.

Satoru Ikehara, Shirai Satoshi and Kentaro Ogura. 1994. Criteria for Evaluating the Linguistic Quality of Japanese-to-English Machine Translation. In Journal of JSAI, 9(5):569-579.

Satoru Ikehara, Shirai Satoshi, Akio Yokoo and Hiromi Nakaiwa. 1991. Toward MT system without Pre-Editing -Effects of New Methods in ALT-J/E. In Proc. of MT Summit $I I I$, pages 101-106.

Megumi Kameyama. 1986. A property-sharing constraint in centering. In 24th Annual Meeting of $A C L$, pages $200-206$.

Atsuo Kawai. 1987. Modality, Tense and Aspect in Japanese-to-English Translation System ALTJ/E. In Proc. of the 34th Annual Convention IPS Japan, pages 1245-1246. In Japanese.

Susumu Kuno. 1978. Danwa no Bunpoo. Taishukan Publ. Co., Tokyo. In Japanese.

Shalom Lappin and Herbert J. Leass. 1994. An Algorithm for Pronominal Anaphora Resolution. In Computational Linguistics, 20(4):535-561, ACL.

Masaaki Murata and Makoto Nagao. 1997. An Estimation of Referents of Pronouns in Japanese Sentence using Examples and Surface Expressions. In 
Journal of Natural Language Processing, 4(1):87109, Association of Natural Language Processing. In Japanese.

Hiromi Nakaiwa and Satoru Ikehara. 1992. Zero Pronoun Resolution in a Japanese-to-English Machine Translation System by using Verbal Semantic Attributes. In Proc. of ANLP92, pages 201208, ACL.

Hiromi Nakaiwa, Akio Yokoo and Satoru Ikehara. 1994. A System of Verbal Semantic Attributes Focused on the Syntactic Correspondence between Japanese and English. In Proc. of COLING94, pages $672-678$.

Hiromi Nakaiwa and Satoru Ikehara. 1995. Intrasentential Resolution of Japanese Zero Pronouns in a Machine Translation system using Semantic and Pragmatic Constraints. In Proc. of TMI95, pages 96-105.

Hiromi Nakaiwa and Satoru Ikehara. 1996. Anaphora Resolution of Japanese Zero Pronouns with Deictic Reference. In Proc. of COLING96, pages $812-817$.

Hiromi Nakaiwa and Setsuo Yamada. 1997. Automatic Identification of Zero Pronouns and their Antecedents within Aligned Sentence Pairs. In Proc. of the 3rd Annual Meeting of the Association for Natural Language Processing. In Japanese.

Tetsuya Nasukawa. 1996. Full-text processing: improving a practical NLP system based on surface information within the context. In Proc. of COLING96, pages $824-829$.

Hideki Tanaka. 1994. Verbal case frame acquisition from a bilingual corpus: Gradual knowledge acquisition. In Proc. of COLING94, pages 727-731.

Marilyn Walker, Masayo Iida and Sharon Cote. 1990. Centering in Japanese Discourse. In Proc. of COLING90.

Setsuo Yamada, Hiromi Nakaiwa, Kentaro Ogura and Satoru Ikehara. 1995. A Method of Automatically Adapting a MT System to Different Domains. In Proc. of TMI95, pages 303-310.

Setsuo Yamada, Hiromi Nakaiwa and Satoru Ikehara. 1996. A New Method of Automatically Aligning Expressions within Aligned Sentence Pairs. In Proc. of NeMLaP2, pages 56-65.

Kei Yoshimoto. 1988. Identifying Zero Pronouns in Japanese Dialogue. In Proc. of COLING88, pages 779-784. 